\title{
An Enhanced Model of Middle Cerebral Artery Occlusion in Nonhuman Primates Using an Endovascular Trapping Technique
}

F.C. Tong, X. Zhang, D.J. Kempf, M.S. Yepes, F.R. Connor-Stroud, S. Zola, and L. Howell

\begin{abstract}
BACKGROUND AND PURPOSE: Current nonhuman primate stroke models are limited by either stroke variability or survivability. A new nonhuman primate stroke model was developed by using endovascular trapping techniques to limit collateral vessels with serial MR imaging and neurologic assessments.
\end{abstract}

MATERIALS AND METHODS: Eight adult rhesus monkeys (female, 7-13 years of age) underwent MR imaging and Spetzler neurologic assessment followed by endovascular stroke induction consisting of superselective endovascular placement of surgical silk sutures into the right MCA by using a trapping technique. Two initial subjects were euthanized immediately following postocclusion MR imaging. The subsequent 6 subjects recovered and underwent follow-up MR imaging and Spetzler neurologic assessments at 48 hours, with 4 being followed to 96 hours. Stroke infarct volumes were measured, and the longitudinal Spetzler clinical neurologic scores were assessed. The brain tissues were harvested and prepared with hematoxylin-eosin staining.

RESULTS: Focal permanent cerebral ischemia was induced in the targeted right MCA territory in all subjects. The volumes of the ischemic lesions at 6, 48, and 96 hours were $3.18 \pm 1.007 \mathrm{~mL}$ (standard error of the mean) $(n=8), 6.70 \pm 1.666 \mathrm{~mL}$ (standard error of the mean) ( $n=$ 6), and $7.23 \pm 1.371 \mathrm{~mL}$ (standard error of the mean) $(n=4)$. For the survival animals, the immediate postsurgical Spetzler grading score improved from 60.7 at 24 hours to 68.7 at 48 hours.

CONCLUSIONS: We report a trapping modification to an established endovascular suture stroke model that yielded reproducible ischemia and clinically quantifiable neurologic deficits with no strokes in nontarget areas. This technique may be useful in evaluating translational stroke and penumbral imaging research in addition to preclinical testing of neuroprotective therapies.

$\mathbf{R}$ odent stroke research models often fail to translate to human stroke. The use of nonhuman primates has advantages in preclinical translational stroke studies. ${ }^{1}$ Gyrencephalic primate species with larger brains demonstrate similar cortical and subcortical organization, with collateral flow that more accurately simulates that in humans. ${ }^{2}$ Early nonhuman primate stroke models used baboons

\footnotetext{
Received December 12, 2014; accepted after revision May 20, 2015.

From the Departments of Radiology and Neurosurgery (F.C.T.), Neurology (M.S.Y.), Psychiatry and Behavioral Sciences (S.Z.. L.H.), School of Medicine, and Yerkes National Primate Research Center (X.Z., D.J.K., F.R.C.-S, S.Z., L.H.), Emory University, Atlanta, Georgia; and Atlanta Veterans Affairs Medical Center (S.Z.), Decatur, Georgia.

This project was supported in part by the National Center for Research Resources and currently by the Office of Research Infrastructure Programs, OD P51OD011132, P51RR000165, DA 031246; and the National Center for Advancing Translational Sciences of the National Institutes of Health under Award Number ULITR000454.

Please address correspondence to Frank C. Tong, MD, Department of Radiology, Emory University Hospital, 1364 Clifton Rd, NE, Atlanta, GA 30322; e-mail: ftong@emory.edu

-- Indicates open access to non-subscribers at www.ajnr.org

http://dx.doi.org/10.3174/ajnr.A4448
}

with transorbital neurosurgical clipping of the middle cerebral artery to address collateral vessels. The resultant strokes involved both the cortex and deep nuclei, requiring prolonged intensive care limiting the opportunity to perform behavioral assessments. ${ }^{3}$

Recent surgical and endovascular stroke models have used nonhuman primates with less robust collaterals and greater similarity to humans, resulting in more consistent stroke results and allowing postoperative neurologic assessments. Approaches include the surgical introduction of an intraluminal thread from the external carotid artery to the origin of the $\mathrm{MCA},{ }^{4}$ injection of autologous clot into the ICA, ${ }^{5}$ injection of silk suture into the $\mathrm{MCA},{ }^{6}$ transient microcatheter occlusion of the $\mathrm{MCA},{ }^{7}$ permanent MCA occlusion with cyanoacrylate, ${ }^{8}$ and combined neurosurgical and endovascular thrombin-injection techniques. ${ }^{9}$ Ideally, a preclinical cortical stroke model would be reproducible, minimally invasive, minimize pain and distress, spare the deep nuclei, and allow serial behavioral assessments. Neurosurgical approaches typically offer more reliable stroke-induction volumes while giving up at least some ability to track neurologic assessments. Endovascular approaches minimize postoperative impair- 
ment but have more stroke volume and distribution variability. We describe a new survivable endovascular trapping technique of ischemic stroke induction in the rhesus monkey (Macaca mulatta), allowing serial behavioral assessment and relative sparing of the deep nuclei.

\section{MATERIALS AND METHODS}

Eight rhesus monkeys were sourced from the Yerkes National Primate Research Center with procedures designed to minimize pain and suffering and approval by the Institutional Animal Care and Use Committee. The subjects were female and ranging from 7 years 9 months to 13 years 7 months and from 6.93 to $9.90 \mathrm{~kg}$. The animals were housed indoors on a standard light-dark cycle individually with routine laboratory diet supplemented by fruits and vegetables with water available at liberty. Two weeks before planned surgery, each subject underwent a routine health and neurologic assessment by using the Spetzler grading scale, ${ }^{10}$ followed by general anesthesia and baseline brain MR imaging.

Two initial animals underwent endovascular stroke induction and serial MR imaging examination with immediate sacrifice. Six subsequent subjects underwent stroke induction, MR imaging scanning, and survival with daily follow-up neurologic assessments and MR imaging at 48 hours (6 of 6 subjects) and 96 hours (4 of 6 subjects). Immediately following the final MR imaging, the animals were sacrificed and the brain tissue was preserved in formalin.

\section{Prescan}

MR imaging prescanning of the brain was performed at least 7 days before stroke induction. Each subject was anesthetized with $3-5 \mathrm{mg} / \mathrm{kg}$ of tiletamine/zolazepam (Telazol) or $10 \mathrm{mg} / \mathrm{kg}$ of ketamine followed by induction of general anesthesia by using $\sim 1.0 \%$ isoflurane mixed with $100 \% \mathrm{O}_{2}$. The pulse rate, respiratory rate, pulse oximetry, end-tidal gas, blood pressure, and endtidal $\mathrm{CO}_{2}$ were monitored and maintained in the normal range. ${ }^{11}$ Body temperature was maintained at $37.5^{\circ} \mathrm{C}$ by a circulating warm-water blanket, and each subject was supine and immobilized with a head holder during scanning. The MR imaging consisted of T1 (magnetization-prepared rapid acquisition of gradient echo sequence with $\mathrm{TR}=2500 \mathrm{~ms}$, $\mathrm{TE}=3.33 \mathrm{~ms}$, FOV $=$ $96 \times 96 \mathrm{~mm}$, flip angle $=8^{\circ}, \mathrm{TI}=950 \mathrm{~ms}$, matrix $=192 \times 192$, section thickness $=1 \mathrm{~mm}, 112$ sections, 1 average), T2 (fast spinecho with $\mathrm{TR}=5000 \mathrm{~ms}, \mathrm{TE}=115 \mathrm{~ms}, \mathrm{FOV}=96 \times 96 \mathrm{~mm}$, $256 \times 256$ matrix, section thickness $=2 \mathrm{~mm}, 2$ averages), DWI (single-shot EPI sequence with generalized autocalibrating partially parallel acquisition acceleration factor $=3$, TR $=5000 \mathrm{~ms}$, $\mathrm{TE}=80 \mathrm{~ms}, \mathrm{FOV}=96 \times 96 \mathrm{~mm}$, data matrix $=64 \times 64$, section thickness $=1.5 \mathrm{~mm}, 30$ directions with $\mathrm{b}$-value $=0,1000 \mathrm{~s} / \mathrm{cm}^{2}$ ), FLAIR $(\mathrm{TR}=10,000 \mathrm{~ms}, \mathrm{TI}=2800 \mathrm{~ms}, \mathrm{TE}=115 \mathrm{~ms}, \mathrm{FOV}=$ $96 \times 96 \mathrm{~mm}$, data matrix $=256 \times 256$, turbo factor $=17$, section thickness $=2 \mathrm{~mm}, 2$ averages $)$, and MRA TOF (FOV $=96 \times 96$ $\mathrm{mm}$, section thickness $=1 \mathrm{~mm}, \mathrm{TR}=39 \mathrm{~ms}, \mathrm{TE}=5.74 \mathrm{~ms}$, single slab $=40$ sections, $448 \times 448$ matrix, single average) on a Magnetom Trio MR imaging scanner (Siemens, Erlangen, German) with an 8-channel phased array knee coil (Invivo Inc. Gainesville, Florida).

\section{Stroke Induction}

The subject was brought to the operating suite, where general anesthesia was administered, and placed in a radiolucent head holder. The bilateral groin regions were shaved, prepped, and draped. Sonographic guidance was used to visualize the right common femoral artery. A small skin nick was placed by using a No. 11 scalpel blade, and a 22-ga access needle (French Silhouette Transitionless Micropunture Introducer Set, Cook Incorporated, Bloomington, Indiana) was placed into the right common femoral artery. This was exchanged over a 0.018-inch Cope Mandrel (Cook Medical) by using a 4F microdilator (Cook Medical). A 4F $65-\mathrm{cm}$ Glide catheter (Terumo, Tokyo, Japan) was placed over a 0.035-inch Bentson guidewire (Olympus, Shinjuku, Japan) by using the Seldinger technique, and $500 \mathrm{U}$ of IV heparin was administered. The 4 French non-taper angle Glide catheter (Terumo; Medical Corporation, Somerset, New Jersey) was placed into the right ICA by using C-arm fluoroscopic guidance (Siremobil Compact, Siemens), and positioning was checked with manual injection of iohexol (Omnipaque 300 contrast; GE Healthcare, Piscataway, New Jersey). Anteroposterior and lateral cerebral angiograms were obtained.

An Echelon 10 (Covidien, Irvine, California) microcather was inserted into the targeted M2/M3 branches of the right MCA under roadmap through the $4 \mathrm{~F}$ diagnostic catheter over a 0.010 -inch SilverSpeed microguidewire (Covidien). Superselective angiography was performed by hand injection to verify suitable placement and stroke targeting. Multiple 5-mm segments of 4-0 silk suture (Ethicon, Johnson \& Johnson, Cincinnati, Ohio) were injected through the microcatheter until slowing of flow within the desired M3 branch was seen. The microcatheter was partially withdrawn into the M1 segment of the right MCA. A final 10-mm segment of suture was injected, and the microcatheter was removed. Anteroposterior and lateral postangiograms were repeated before catheter removal and administration of $2 \mathrm{mg}$ of protamine sulfate IV. Hemostasis was obtained with manual compression for 15 minutes and a single 4-0 polydioxanone suture (PDS Plus Sutures; Ethicon).

\section{Poststroke Imaging, Neurologic Assessment, and Recovery}

Poststroke images were repeated up to 7 hours after stroke induction. The 2 initial subjects were then euthanized, while the subsequent 6 subjects recovered from anesthesia and were monitored. The survival subjects were maintained in an individual cage with veterinary staff supervision and 24-hour continuous video monitoring. Softened routine veterinary chow, fruit, and water were offered. Assessment of overall well-being and Spetzler neurologic deficit scoring were performed daily, with MR imaging scans repeated at 48 hours ( 6 of 6 subjects) and 96 hours ( 4 of 6 subjects) poststroke. Two of the 6 survival subjects were sacrificed following the 48-hour scanning at the recommendation of the veterinary staff. MR images were reviewed, and stroke volumes were calculated from DWI by using a threshold of the mean \pm 2 SDs with the contralateral side as a reference. Lesion volumes from DWI were derived from the threshold (mean \pm 2 SDs) of the DWI intensity on the contralateral side by using custom Matlab scripts (MathWorks, Natick, Massachusetts). Statistical analysis of the 

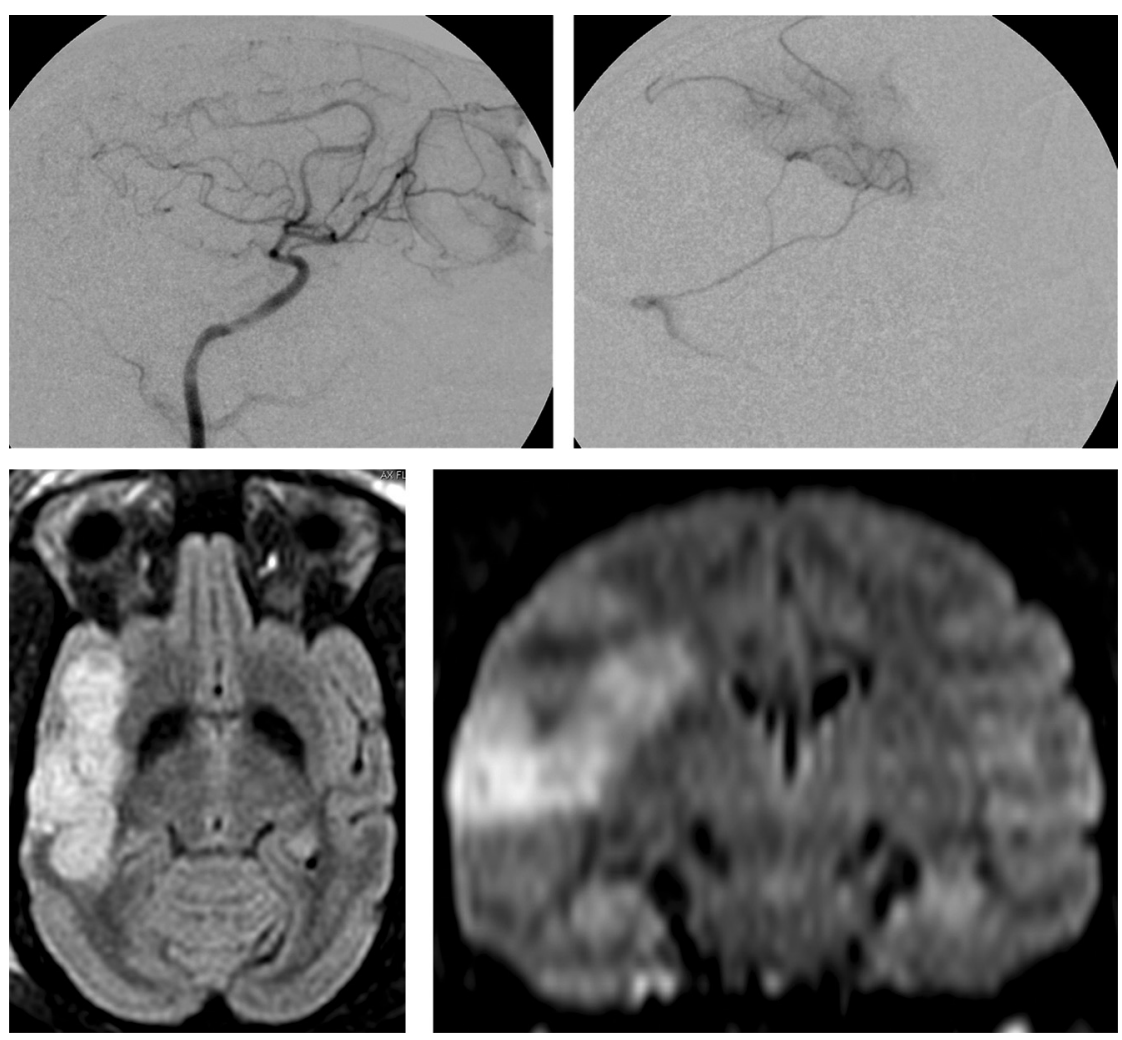

FIG 1. Upper left: lateral angiogram from right internal carotid artery injection. Upper Right: superselective microcatheter angiogram in the right M3 branch. Lower Left: 96-hour FLAIR image showing cortical infarct. Lower Right: 96-hour reconstructed coronal FLAIR image showing cortical infarct with sparing of the basal ganglia.

lesion volumes was performed for the 3 time points by using unpaired 2-tailed $t$ tests.

\section{Sacrifice and Histology}

After the 96-hour scan, the subjects were euthanized immediately while still under general anesthesia by using IV pentobarbital, $100 \mathrm{mg}$, and transcardial perfusion with saline solution and $10 \%$ buffered formalin following the approved protocols of the Emory Institutional Animal Care and Use Committee. The brains were harvested, immersed in $10 \%$ buffered formalin, sectioned onto $50-\mu \mathrm{m}$ sections, and stained with the hematoxylin-eosin technique.

\section{RESULTS}

\section{Stroke Induction}

Angiograms demonstrating microcatheter placement during the stroke-induction procedure and resultant MR imaging are shown in Fig 1. All 8 subjects had new strokes in the targeted right MCA territories on the postoperative MR images. Each of the 6 designated survival animals recovered from anesthesia successfully. Immediate and delayed MR imaging showed no stroke or emboli in unintended territories or hemorrhage in any of the subjects.

\section{MR Imaging, Stroke Volumes, and Histology}

Stroke volumes were calculated from the 6-, 48-, and 96-hour DWI scans and are summarized in Table 1. The average stroke volume at 6 hours was $3.18 \mathrm{~mL}(n=8$; range, $0.5-8.8 \mathrm{~mL})$ for all 8 subjects. For the 6 survival subjects, the average stroke volume was $6.70 \mathrm{~mL}$ at 48 hours ( $n=6$; range, $3.0-12.9 \mathrm{~mL}$ ) and $7.23 \mathrm{~mL}$ at 96 hours ( $n=4$; range, $3.3-9.5 \mathrm{~mL}$ ). For the 4 subjects that survived to 96 hours, the 48-hour average infarct volume was 6.0 $\mathrm{mL}$ ( $n=4$; range, $3.0-10.0 \mathrm{~mL}$ ). Infarct volume difference reached statistical significance from 6 to 96 hours $(P=.041$; 95\% CI, 0.193-7.882) but not from 6 to 48 hours or from 48 to 96 hours. Representative coronal FLAIR images and the corresponding anatomic sections are shown in Fig 2.

\section{Complications}

Technical Complications. There were no procedural complications encountered in this series. Specifically, there were no vessel perforations, no unintended embolic stroke, no intracranial hemorrhage, no vessel dissection, and no groin complications.

Clinical Complications/Early Clinical Sacrifice. Two subjects were sacrificed immediately following the 48-hour scan at the recommendation of the veterinary staff in accordance with the end points approved by Institutional Animal Care and Use Committee. RPF6 had a relatively small right basal ganglia and insular stroke and started to manifest seizure activity before the 48 -hour scan and was subsequently euthanized immediately after the 48 -hour scan. RJJ3 was also euthanized after the 48-hour scan with symptoms of hemineglect, left hemiparesis, and gaze deviation while lying recumbent on the right side unable to maintain upright posture without assistance.

\section{Cognitive Evaluation/Neurologic Assessment}

Formal daily neurologic assessments were performed by using the Spetzler neurologic deficit scoring scale shown in Table 2, ranging from 1 to 100 with 70 points for motor function, 20 points for behavior (awareness and aggression), 5 points for visual fields, and 5 points for cranial nerve function. The average scores of the 6 survival subjects are shown in Table 3 and were 60.7 at 6 hours and 68.7 at 48 hours, with the most common score deductions for contralateral motor weakness. With euthanasia of 2 subjects at 48 hours, the remaining 4 subjects that survived to 96 hours had an average neurologic score of 85 at 48 hours (range, 85-85) and 84 at 96 hours (range, 81-84).

\section{DISCUSSION}

\section{Collateral Flow and Stroke Models}

One of the challenges in using nonhuman primates for modeling human stroke is that the degree of collateral circulation is often more robust compared with human circulation. ${ }^{2,12}$ This difference makes the nonhuman primate brain more resistant to stroke 
induction, requiring temporary or permanent elimination of collateral vessels. Published series of stroke and temporary ischemia models have used open surgical, endovascular, or mixed techniques, ${ }^{4,9,13-16}$ many targeting collateral vessels as a means of decreasing cerebral flow. Management of collateral vessels is critical in achieving cerebral blood flow below the infarct threshold of approximately $0.12 \mathrm{~mL} / \mathrm{g}$ per minute. While values above this threshold may result in cessation of function, flow rates below this level are required to achieve cell death within the targeted region. ${ }^{17}$ With this requirement in mind, our study used older subjects that could potentially have fewer collateral vessels; fewer col-
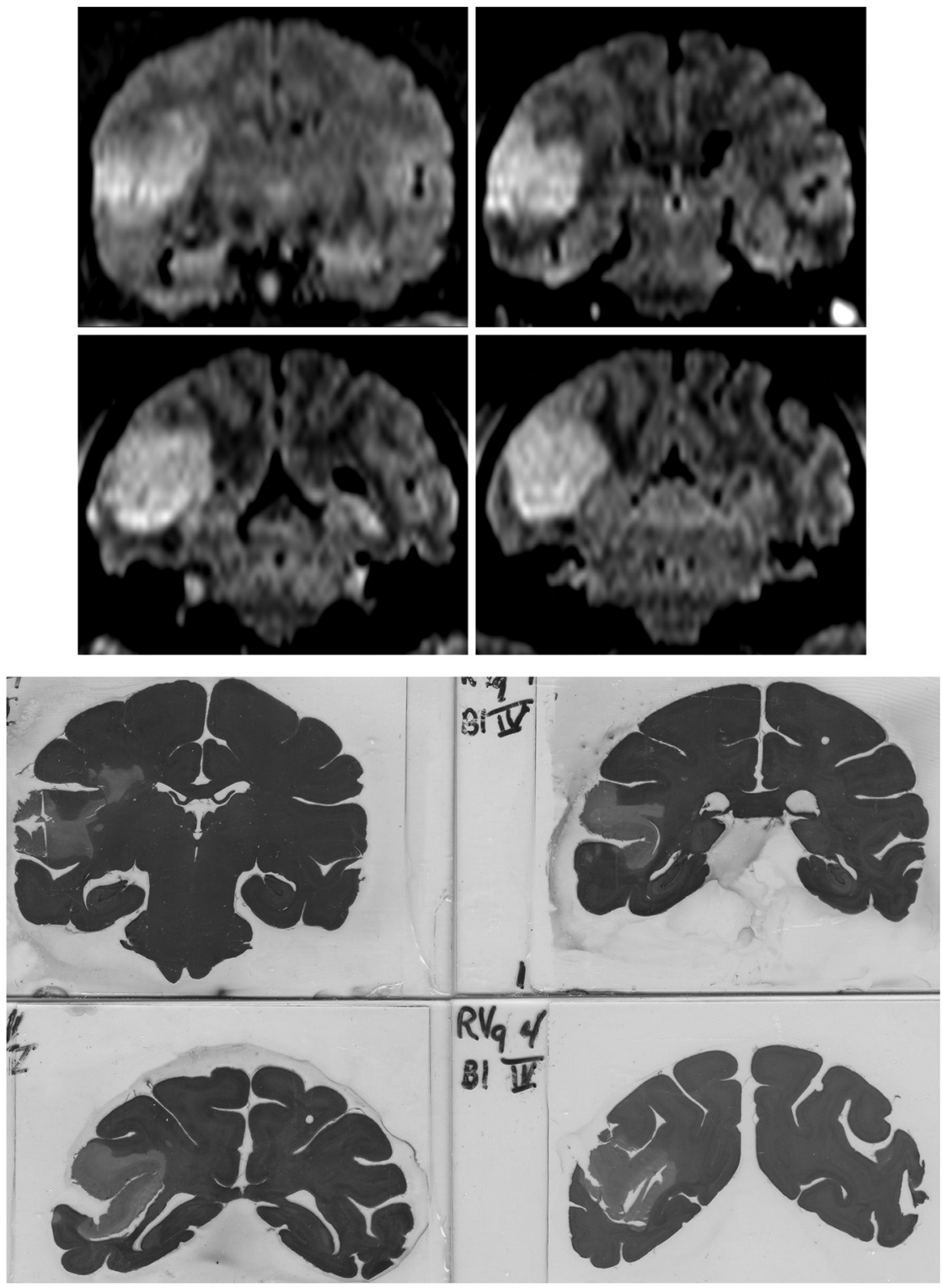

FIG 2. Upper: coronal FLAIR images showing cortical infarction at 96 hours. Lower: corresponding hematoxylin-eosin-stained anatomic sections following sacrifice. lateral vessels have been shown in both aging mice ${ }^{18}$ and humans with proximal MCA occlusion. ${ }^{19}$

Hudgins and Garcia ${ }^{20}$ published an approach in 1970 consisting of orbital enucleation followed by MCA microclip placement to regulate the degree of cerebral ischemia. This method is still used, with the primary drawback being its invasive nature and disallowance of thrombolytics used in current stroke therapy. Another approach used temporary clipping of the bilateral anterior cerebral arteries and MCA for reducing collateral flow, ${ }^{12}$ and this approach yielded relatively consistent cortical stroke volumes of approximately $25 \%$ of the entire cortical volume. Comparatively, our method is less invasive and avoids the MR imaging artifacts due to surgical clip placement.

A purely endovascular reversible stroke model was published by Jungreis et $\mathrm{al}^{21}$ by using permanent coiling of the right posterior cerebral artery and temporary balloon occlusion at the junction of the ICA terminus, anterior cerebral artery, and MCA. The reversible nature of this MCA-occlusion model used permanent coil occlusion of the posterior cerebral artery, and the authors did not comment on the actual stroke volumes or survivability. A subsequent study using this model resulted in relatively large infarct volumes with involvement of the basal ganglia in a small nonsurvival study. ${ }^{22}$ Our approach offers the advantage of basal ganglia sparing in 7 of 8 subjects and stroke volumes better suited to survivability studies.

Other endovascular models used temporary mechanical catheter occlusion, injection of $n$-butyl-cyanoacrylate or other permanent agents. ${ }^{8}$ One disadvantage of these approaches is again inconsistent lesion sizes, though it may reflect similar variation in human stroke. Our endovascular approach resulted in stroke in the targeted territory in $100 \%$ of subjects with $100 \%$ 48hour survivability without procedural complications or unintended strokes. However, 2 of the intended survival subjects were euthanized for compassionate rather than medical reasons at the recommendation of the supervising veterinarian.

Table 1: Experimental infarct volumes (cc) estimated from the diffusion-weighted images at various time intervals postocclusion

\begin{tabular}{lccccccccc}
\hline Stroke Timing & RVI3 & RCE3 & RFP6 & RJJ3 & RFA5 & RRI3 & PH1019 & RVG4 & Average ( \pm SEM) \\
\hline 6 Hours & 3.3 & 8.8 & 0.6 & 4.6 & 4.9 & 1.6 & 0.5 & 1.2 & $3.18 \pm 1.007$ \\
48 Hours & & & 3.3 & 12.9 & 10.0 & 3.0 & 7.2 & 3.8 & $6.70 \pm 1.666$ \\
96 Hours & & & & & 9.5 & 3.3 & 8.6 & 7.5 & $7.23 \pm 1.371$ \\
\hline
\end{tabular}

Note:-SEM indicates standard error of the mean; RV13, RCE3, RFP6, RJJ3, RFA5, RR13, PH1019, RVG4, rhesus monkey subjects. 


\section{Stroke Induction and Survivability}

RPF6 was the first subject in the survival cohort and developed a small stroke in the right basal ganglia. Postrecovery, the subject developed clinical seizure activity just before the 48-hour MR imaging scan and was euthanized immediately after the scan. The imaging would suggest that the basal ganglia stroke location was the most likely source of the seizure activity. While this type of stroke is not uncommonly seen and routinely supported in human patients, the ability to care for the nonhuman primate in the absence of an intensive care unit-type environment necessitated early sacrifice. Clinically, this was not unexpected because the overall rate of poststroke seizure in human patients ranges from $4.4 \%$ to $11.1 \%,{ }^{23,24}$ with approximately $3.1 \%{ }^{25}$ developing poststroke seizure within the first 24 hours. Subsequently, the endovascular technique was modified slightly to spare the basal ganglia in the remaining 5 survival subjects, and no additional basal ganglia strokes were observed.

The other 5 survival subjects had purely cortical strokes without basal ganglia involvement and manifested seizure activity with progression of stroke volumes. One survival subject was euthanized for compassionate reasons for symptoms of generalized lethargy and diminished oral intake (an end point approved by Institutional Animal Care and Use Committee). Clinically, the relatively large stroke in this subject is commonly seen and treated in human patients; $15 \%-20 \%$ of patients require hospital care in the intensive care unit. ${ }^{26}$ The remaining 4 survival subjects tolerated their strokes well and demonstrated consistent clinical grades on the Spetzler grading scale. There was a trend toward increasing infarct size between the 6- to 48-hour scans and the 48- to 96-hour

Table 2: Spetzler neurologic deficit scoring

\begin{tabular}{lr}
\hline \multicolumn{1}{c}{ Scoring } & \\
\hline Motor function & 10 \\
Severe hemiparesis & 25 \\
Mild hemiparesis & 55 \\
$\quad$ Normal strength: favors opposite extremity & 70 \\
Normal strength: normal function & \\
Behavior & 0 \\
Death & 1 \\
Coma & 5 \\
Aware of surroundings: not active & 15 \\
Aware of surroundings: moves in response to examiner & 20 \\
Normal aggression & \\
Ocular and cranial nerve function & 1 \\
Facial movement: paretic & 5 \\
Facial movement: normal & 1 \\
Visual field: hemianopic & 5 \\
Visual field: normal & \\
\hline
\end{tabular}

scans, with only the 6- to 96-hour infarct volume reaching statistical significance. Overall, there was no symptomatic worsening in clinical examination findings from 24 to 48 hours for the survival arm of the study. At the 96-hour mark, the remaining subjects were in no clinical distress, which suggests that they could likely have potentially survived for a longer period. Given our experimental and clinical results, longer survival periods would be facilitated by avoidance of basal ganglia strokes in addition to very large stroke volumes.

\section{Endovascular Trapping and Stroke Accuracy}

Embolic silk suture has been described in the treatment of brain AVMs and does not cause significant inflammatory change, making it suitable for use in a permanent stroke model. ${ }^{27,28}$ It is deliverable through a microcatheter, is MR imaging-compatible, and has been previously used in the nonhuman primate model, with the injection of very short segments of silk from the proximal MCA with variable clinical and imaging results. ${ }^{6}$ Comparatively, our superselective trapping method results in better survivability and more controlled stroke volumes and localization.

Each of the 8 experimental strokes had positive DWI changes located precisely between the selected distal M3 branch and the pullback proximal M1 position of the endovascularly placed microcatheter. Although more technically demanding, placement of the smaller 5-mm suture segments into the distal M3 segment of the MCA effectively eliminates retrograde collateral flow in the MCA branch. Similarly, placement of the proximal $10-\mathrm{mm}$ suture effectively traps the desired MCA segment more precisely than if the suture were injected from the proximal MCA segment.

The need to spare the lenticulostriate arteries was shown by the basal ganglia stroke/seizure in the first survival subject. The endovascular trapping technique was subsequently enhanced with no additional basal ganglia strokes in the remaining 5 survival subjects. First-time seizures occur at a rate of up to $15 \%$ in patients with clinical stroke, ${ }^{29}$ so it is not unexpected for seizures to occur in our series. However, it is not practical to care for these types of seizures in the animal care environment, and the subject was euthanized to preserve its comfort in compliance with the Institutional Animal Care and Use Committee guidelines. Similarly, the second subject showed symptoms of generalized lethargy and diminished oral uptake that would be within the expected scope of human stroke intermediate level care. Our results suggest enhanced accuracy and stroke yield with better basal ganglia sparing.
Table 3: Survival subject neurologic score

\begin{tabular}{|c|c|c|c|c|c|c|}
\hline Subject & Baseline & 24 Hours & 48 Hours & 96 Hours & $\begin{array}{l}\text { Basal Ganglia } \\
\text { Involvement }\end{array}$ & Sxs \\
\hline RFP6 & 100 & 46 & 51 & & Yes & Seizures \\
\hline RJJ3 & 100 & 21 & 21 & & No & $\begin{array}{c}\text { Severe hemiparesis, } \\
\text { hemianopsia }\end{array}$ \\
\hline RFA5 & 100 & 46 & 85 & 85 & No & \\
\hline RRI3 & 100 & 81 & 85 & 85 & No & \\
\hline PH1019 & 100 & 85 & 85 & 85 & No & \\
\hline RVG4 & 100 & 85 & 85 & 81 & No & \\
\hline Mean & 100 & 60.7 & 68.7 & 84 & & \\
\hline
\end{tabular}

Note:-Sxs indicates major symptoms

\section{CONCLUSIONS}

Our endovascular trapping modification of an existing suture-injection stroke model allows rapid poststroke recovery. Accurate neurologic assessments can be performed immediately in a noncritical care setting. This assessment results in improved survivability, basal ganglia sparing, and consistency compared with previously published endovascular methods. Furthermore, 
translational thrombolytic or neuroprotective therapies can be evaluated; these evaluations are not possible with open neurosurgery and the resulting confounding postoperative factors. Endovascular trapping offers a greater degree of precision for stroke location and size. Although 1 limitation of our model is that it uses a permanent agent, it could be easily adapted to a temporary occlusion model if a suitable injectable agent were identified.

\section{ACKNOWLEDGMENTS}

The authors recognize the contributions of Sudeep Patel, Chunxia Li, Govind Nair, Yumei Yan, Ruth Connelly, Wendy Williamson Coyne, Juliet Brown, Anapatricia Garcia, E. Chris Muly, and Marcelia Maddox.

\section{REFERENCES}

1. Stroke Therapy Academic Industry Roundtable (STAIR). Recommendations for standards regarding preclinical neuroprotective and restorative drug development. Stroke 1999;30:2752-58 CrossRef Medline

2. Cook DJ, Tymianski M. Nonhuman primate models of stroke for translational neuroprotection research. Neurotherapeutics 2012;9: 371-79 CrossRef Medline

3. Nehls DG, Cartwright M, Spetzler RF. Experimental primate stroke model. Neurosurgery 1986;18:388-89 CrossRef Medline

4. Freret $\mathrm{T}$, Bouet $\mathrm{V}$, Toutain J, et al. Intraluminal thread model of focal stroke in the non-human primate. J Cereb Blood Flow Metab 2008; 28:786-96 CrossRef Medline

5. Yoshikawa T, Akiyoshi Y, Susumu T, et al. Ginsenoside Rb1 reduces neurodegeneration in the peri-infarct area of a thromboembolic stroke model in non-human primates. J Pharmacol Sci 2008;107: 32-40 CrossRef Medline

6. Rodriguez-Mercado R, Ford GD, Xu Z, et al. Acute neuronal injury and blood genomic profiles in a nonhuman primate model for ischemic stroke. Comp Med 2012;62:427-38 Medline

7. de Crespigny AJ, D'Arceuil HE, Maynard KI, et al. Acute studies of a new primate model of reversible middle cerebral artery occlusion. $J$ Stroke Cerebrovasc Dis 2005;14:80-87 CrossRef Medline

8. Liu Y, D'Arceuil HE, Westmoreland S, et al. Serial diffusion tensor MRI after transient and permanent cerebral ischemia in nonhuman primates. Stroke 2007;38:138-45 CrossRef Medline

9. Gauberti M, Obiang P, Guedin P, et al. Thrombotic stroke in the anesthetized monkey (Macaca mulatta): characterization by MRI-a pilot study. Cerebrovasc Dis 2012;33:329-39 CrossRef Medline

10. Spetzler RF, Selman WR, Weinstein P, et al. Chronic reversible cerebral ischemia: evaluation of a new baboon model. Neurosurgery 1980;7:257-61 CrossRef Medline

11. Li CX, Patel S, Auerbach EJ, et al. Dose-dependent effect of isoflurane on regional cerebral blood flow in anesthetized macaque monkeys. Neurosci Lett 2013;541:58-62 CrossRef Medline

12. West GA, Golshani KJ, Doyle KP, et al. A new model of cortical stroke in the rhesus macaque. J Cereb Blood Flow Metab 2009;29: 1175-86 CrossRef Medline

13. Cui Y, Takamatsu H, Kakiuchi T, et al. Neuroprotection by a central nervous system-type prostacyclin receptor ligand demonstrated in monkeys subjected to middle cerebral artery occlusion and reperfusion: a positron emission tomography study. Stroke 2006;37: 2830-36 CrossRef Medline

14. Nudo RJ, Larson D, Plautz EJ, et al. A squirrel monkey model of poststroke motor recovery. ILAR J 2003;44:161-74 CrossRef Medline

15. Chin Y, Sato Y, Mase M, et al. Transient decrease in cerebral motor pathway fractional anisotropy after focal ischemic stroke in monkey. Neurosci Res 2010;66:406-11 CrossRef Medline

16. Del Zoppo GJ, Copeland BR, Harker LA, et al. Experimental acute thrombotic stroke in baboons. Stroke 1986;17:1254-65 CrossRef Medline

17. Sakoh M, Ostergaard L, Rohl L, et al. Relationship between residual cerebral blood flow and oxygen metabolism as predictive of ischemic tissue viability: sequential multitracer positron emission tomography scanning of middle cerebral artery occlusion during the critical first 6 hours after stroke in pigs. J Neurosurg 2000;93: 647-57 CrossRef Medline

18. Faber JE, Zhang H, Lassance-Soares RM, et al. Aging causes collateral rarefaction and increased severity of ischemic injury in multiple tissues. Arterioscler Thromb Vasc Biol 2011;31:1748-56 CrossRef Medline

19. Arsava EM, Vural A, Akpinar E, et al. The detrimental effect of aging on leptomeningeal collaterals in ischemic stroke. J Stroke Cerebrovasc Dis 2014;23:421-26 CrossRef Medline

20. Hudgins WR, Garcia JH. Transorbital approach to the middle cerebral artery of the squirrel monkey: a technique for experimental cerebral infarction applicable to ultrastructural studies. Stroke 1970;1:107-11 CrossRef Medline

21. Jungreis CA, Nemoto E, Boada F, et al. Model of reversible cerebral ischemia in a monkey model. AJNR Am J Neuroradiol 2003;24: 1834-36 Medline

22. Kharlamov A, LaVerde GC, Nemoto EM, et al. Map2 immunostaining in thick sections for early ischemic stroke infarct volume in non-human primate brain. J Neurosci Methods 2009;182:205-10 CrossRef Medline

23. Graham NS, Crichton S, Koutroumanidis M, et al. Incidence and associations of poststroke epilepsy: the prospective South London Stroke Register. Stroke 2013;44:605-11 CrossRef Medline

24. Wang G, Jia H, Chen C, et al. Analysis of risk factors for first seizure after stroke in Chinese patients. Biomed Res Int 2013;2013:702871 CrossRef Medline

25. Szaflarski JP, Rackley AY, Kleindorfer DO, et al. Incidence of seizures in the acute phase of stroke: a population-based study. Epilepsia. 2008;49:974-81 CrossRef Medline

26. Zazulia AR. Critical care management of acute ischemic stroke. Continuum Lifelong Learning in Neurology 2009;15:68-82 CrossRef

27. Schmutz F, McAuliffe W, Anderson DM, et al. Embolization of cerebral arteriovenous malformations with silk: histopathologic changes and hemorrhagic complications. AJNR Am J Neuroradiol 1997;18:1233-37 Medline

28. Dehdashti AR, Muster M, Reverdin A, et al. Preoperative silk suture embolization of cerebral and dural arteriovenous malformations. Neurosurg Focus 2001;11:e6 Medline

29. Velioğlu SK, Ozmenoğlu M, Boz C, et al. Status epilepticus after stroke. Stroke 2001;32:1169-72 CrossRef Medline 